\title{
Modelling of Supply Chain Risk Contagion Based on System Dynamics
}

\author{
Dingfu Jiang*, Caihong Liu \\ Department of Business, Jiaxing University, Jiaxing 314001, China
}

Corresponding Author Email: jiangdingfu@ sina.com

https://doi.org/10.18280/jesa.520207

Received: 12 January 2019

Accepted: 20 March 2019

\section{Keywords:}

supply chain (SC), risk contagion, system

dynamics, evolution

\begin{abstract}
In view of the risk contagion in supply chain (SC) regarded as a complex and non-linear system and its severe threat to the entire SC system, it is very necessary for the research on risk contagion of SC. Based on the analysis of risk contagion process for SC and the theory of system dynamics, from the perspective of the risk management decision-making behavior of enterprise in SC, this paper creatively constructs the risk contagion model. Next, simulation for this model was carried out to explore the evolution and mechanism of SC risk contagion, and reveal the risk contagion effects of individual enterprises on SC system. The results show that enterprises with different risk resistances have different abilities to control risk contagion, and need to start risk control under different conditions. Thus, the longer the incubation period of the SC risk, the more harmful the risk contagion for the three types of enterprises. At the same time, enterprises with different risk resistance and risk control capabilities, the contagion effect of risk in the three types of enterprises are different, enterprise should develop different strategies for risk management decisions at different times. Moreover, for enterprises in SC, enhancing their risk immunity is far more important than simply emphasizing risk control technology. On some extent, the research findings can help risk decision-makers verify the feasibility of risk control strategies, also help enterprises to analyze the contagious risks being faced, and to take effective measures to avoid those problems. It can also provide some reference to scholars for the related study.
\end{abstract}

\section{INTRODUCTION}

With the deepening of global integration and refinement of division of labor, enterprises are increasingly dependent on other members in the supply chain (SC). Meanwhile, the SC network has evolved into a complex and specialized system to satisfy the requirements of global procurement, dynamic alliance, rapid response and information sharing. In this process, the $\mathrm{SC}$ system becomes more and more vulnerable to external influence. Since the 1990s, supply chain (SC) emergencies have occurred at a growing frequency, owing to the dynamic market development and diversified personal demand. These emergencies have brought huge losses to many enterprises. For example, the 2016 Kumamoto earthquakes delivered a heavy blow to the automotive industry in Japan. In the same year, the floods in Louisiana forced the closure of the fourth largest refinery in the US. The Hurricane Maria in 2017 also evoked lasting negative repercussions on SCs. As far as China is concerned, the GDP growth, coupled with the boom of import/export, has exposed SCs to rapid growing risks. The $\mathrm{SC}$ emergencies may arise from natural disasters, equipment failures and other conventional factors. A typical example is the 2018 ZTE incident, which is resulted from the US ban on chip supply to the ZTE, a Chinese multinational telecommunications equipment and systems company.

In an SC system, the risk generated at a node can immediately spread to the adjacent enterprises in the up- and downstream, and then propagate across the entire SC through the relational structure. This phenomenon, often known as risk contagion, poses a major threat to the SC security. The highly contagious risk may accumulate, amplify, and event mutates, and eventually develops into a crisis, causing the interruption or failure of the SC system. As a result, many scholars have explored the risk contagion between $\mathrm{SC}$ enterprises, aiming to build a SC network that can effectively control risk contagion. In general, the following three aspects have attracted the most attention: the risk contagion mechanism [1, 2], the risk contagion effects $[3,4]$ and the risk contagion models [5-8]. However, the relevant studies have not formed a systematic research framework or approach. Neither have they provided powerful tools to contain SC risk contagion. This calls for further research into the mechanism and effect of SC risk contagion, especially the risk source and contagion path. The research results may of great help to create targeted measures against SC risk contagion. Of course, it is immensely difficult to develop a highly reliable and universal strategy for risk contagion control, for the SC system has been greatly complicated by the dynamic global market, refined division of labor and the interdependence between SC nodes. Despite all these, it may be possible to disclose the rules and features of SC risk contagion, and mitigate the losses induced by risk contagion, from the perspective of the macro-network structure and micro-agent behavior.

Based on the theory of system dynamics, this paper analyzes the core factors and contagion behavior of SC risks, and develops a model on the risk contagion process in the SC system. Next, simulation analysis was carried out to explore the evolution and mechanism of SC risk contagion, and reveal how risk contagion affects the SC system and individual enterprises. The research findings shed new light on the formulation of rational risk control strategies. 


\section{SC RISK CONTAGION ANALYSIS}

\subsection{The concept of SC risk contagion}

Traditionally, risk is defined as the possibility and consequence of an uncertain event, which deviates from the expected goal of the decision maker. This deviation is usually used as an indicator to measure the extent of risk [9]. At present, some scholars try to use the concept of risk to defining the supply chain risk, but in view of Supply chain system as a complex system, its risk is difficult to be defined. Usually, the SC risk has been defined differently, depending on some perspectives $[10,11]$. Nevertheless, all definitions of supply chain risk are based on the concept of risk, so the core content is relatively consistent, the main points are as follows [12]: (1) The uncertainty factors in SC are the main sources of SC risk. (2) Supply chain risk can lead to Bullwhip effect, which enlarges the risk utility of supply chain (i.e. supply chain risk is contagious and diffused). (3) Supply chain risk is characterized by its vulnerability.

Due to the interdependence of among enterprises in SC, any problem in an enterprise will magnify its risk in SC under the Bullwhip Effect. In recent years, the catastrophic consequences of risk events in SC have made SC risk contagion an urgent research topic. In SC system, the risk of one enterprise may spread to the enterprises in the up- or downstream. With the elapse of time, the risk accumulates, amplifies and mutates until it disrupts the supply or demand will eventually evolve into a crisis, causing interruption or failure of the SC. But, similarly, there is also no clear, direct definition of SC risk contagion. Sometimes, this concept is described in other names (e.g. SC risk conduction, SC risk transmission), which have exactly the same meaning. Such as, drawing on the physical meaning of transmission, Qiu [13] defined SC risk transmission as the diffusion of the risk from the source to SC enterprises, under the effect of the risk booster, through the connections between the enterprises, and suggested that this process prevents the SC system to acquire the expected benefits. Li [14] gave the following definition to $\mathrm{SC}$ risk conduction: under the unavoidable interference and influence of internal and external uncertainties, the SC risk permeates the production and operation of SC enterprises in various forms, making them unable to achieve the production or operation goals. Reference [15] describes the risk contagion phenomenon as the propagation of the SC risk along the SC structure to the upstream and downstream enterprises, creating threats or losses to other SC members and leading to the breakdown of the SC.

$\mathrm{SC}$ risk contagion is sudden and concealed. The risk can spread to relevant SC nodes at a high speed, resulting in serious consequences. The failure of any SC node may trigger a violent chain reaction, which ends up as the interruption or failure of the entire SC system [16]. Hence, our research on SC risk contagion effect has theoretical and practical significance for the healthy and sound development of SCs.

\subsection{SC risk contagion process}

Each node in the SC system has some uncertainties, due to the interaction between internal and external environments. Once a risk catalyst takes effect, the risk of a node may become the source of risk contagion. Risk source is the basis of risk transmission in SC [17]. In this paper, the risk contagion process is explored from the perspective of enterprises, that is, the evolution of the risk of a single enterprise into the risk of the entire SC system.

There are many types of SC risks, ranging from reputation risk, information risk, and technology risk to management risk, etc. These risks can be attributed to the contradiction between demand and supply. Therefore, it is assumed that the failure to meet consumer demand is to fundamental cause of SC risks. This means demand satisfaction must be considered in the analysis of SC risk contagion.

Besides, the risks are not transmitted in the same quantity or size in the contagion process. Instead, the SC enterprises suffer damages of different intensities, depending on the power of the risks and their abilities to manage and eliminate risks. As a result, the probability of each enterprise being infected by risk should also be considered in the evaluation of the enterprise's risk resistance.

In addition, the risk contagion is nonlinear and bidirectional. On the one hand, the direct or indirect propagation of risks depends on the relational network between SC enterprises, which belong to different levels. On the other hand, the SC enterprises have mutual influences on each other, forming a complex networked structure. Through the structure, risks can transfer, accumulated and amplified among SC enterprises through business flow, logistics flow, capital flow and information flow, which are actually the carriers of risk, that is, the transmission medium of risk.

According to the risk susceptibility, risk receivers can be divided into three types: strong relationship enterprises, weak risk resistance enterprises and enterprises with low risk tolerance. The latter two have poor resistance to risk. The former enterprise has strong resistance to risk because of its flexible resource integration ability.

Based on the above understanding, this paper prepares a flowchart of SC risk contagion (Figure 1).

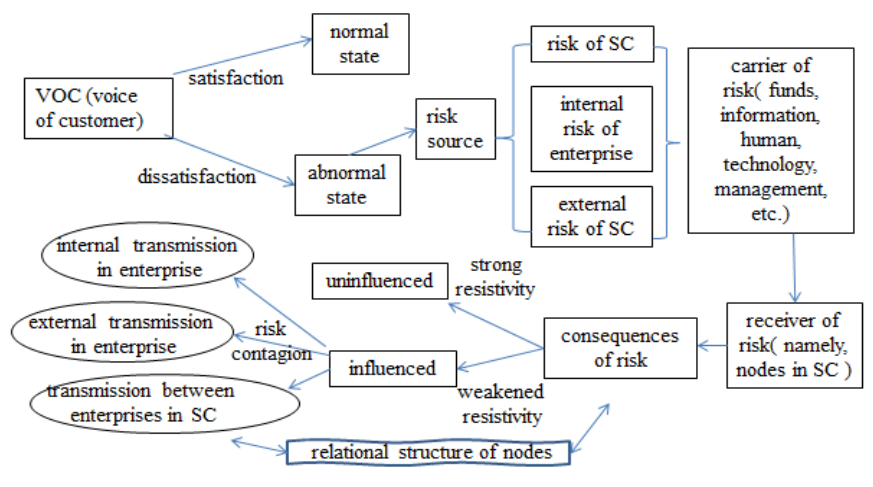

Figure 1. Flowchart of SC risk contagion

It can be seen from Figure 1 that, after the initiation of SC risk contagion, the enterprises of strong correlation, weak risk tolerance and poor risk resistance are the most likely to be affected. Once affected, these enterprises will spread the risk to their affiliates. By contrast, the enterprises of weak correlation, strong risk tolerance and good risk resistance are much less likely to be affected, and can take active measures to control the risk and reduce the losses. Through the contagion process, the SC risk will evolve from the risk of a single enterprise to the risk affecting the entire SC system. This means the mechanism of SC risk contagion is the propagation from the risk source to the risk receivers. 


\section{SC RISK CONTAGION MODELLING BASED ON SYSTEM DYNAMICS}

\subsection{Risk sources}

The consumers in the SC environment fall into two major categories: terminal users and enterprises. The terminal users are concerned about the demands of personalized products and services, while the enterprises face three kinds of problems concerning resource demand, namely, upstream problems (e.g. the supply of raw materials), internal problems (e.g. production and management) and downstream problems (e.g. sales).

Therefore, the SC risk mainly refers to the possible events in the SC that may lead to dissatisfaction of products and services, that is, the SC risk comes from unmet consumer demand. Among the risk carriers in Figure 1, products and services are the fundamental ones, serving as the basis of other risk carriers. Comparatively, the SC risk is more likely to originate from products than services. In many cases, product risk is used as the byname of the SC risk. Note that product risk is not limited to the function of products, but exists in every stage of the $\mathrm{SC}$, from $\mathrm{R} \& \mathrm{D}$, procurement, production to sales. In other words, product quality faces the risks from every aspect in the SC environment, ranging from production to sales. Meanwhile, service risk mainly concerns the quality of information about the products and the provision of valueadded services.

In view of the fact that the source of this risk lies in product quality, service quality and enterprise's own risk management ability, in this paper, the SC risk contagion are explored based on the following sources: the SC risk hinges on products, services and risk control ability; the quality of products and services is evaluated by consumers; the quality of risk control is reflected in the control effect related to enterprise risk management decision-making ability. On this basis, this paper measures the SC risk against two criteria, namely, consumer satisfaction, and risk control effect, which depends on the risk management decision-making behavior of enterprises. This research perspective helps to identify the SC risk accurately, and realize macro-control of adverse events.

\subsection{Hypotheses}

For simplicity, the following hypotheses were put forward:

(1) From the perspective of manufacturing enterprises, the SC risk has four possible sources (product, service, resource supply and demand, and information), regardless of natural and political factors. The information entropy of risk in each source can be determined separately according to the relevant influencing factors. Then, the four entropies can be synthetized into the information entropy of the SC risk.

(2) From the perspective of the SC structure, the SC risk is incentivized by structure robustness, logistical effectiveness, cooperation strength and market adaptability. These incentives can interact with the four possible sources in Hypothesis (1). In fact, there is a mutual dependence between the risk factors of the SC structure and those of manufacturing enterprises (in the supply and demand process).

(3) Each SC node has certain requirements on supply or demand. When the supply or demand requirements of every node are fulfilled, the corresponding SC state can be taken as the ideal situation.
(4) The supply and demand flows in the SC are multi-level and bidirectional.

\subsection{SC risk contagion modelling}

\subsubsection{Complex SC system}

The SC system is the entirety of numerous members (e.g. suppliers, manufacturers, distributors and consumers), the correlation of each member with others and the external environment, various business activities (e.g. forecast, planning and distribution) and multiple flows (e.g. information, material and capital). The complex structure of the SC and the intricate interaction between SC members give rise to many problems in SC management, including but not limited to the bullwhip effect, contract management, cost control, and coordination with the environment.

The above analysis shows that the SC system is essentially a giant complex system, similar to the weather system [18 20]. The system complexity must be fully considered to identify the concealed nonlinear features and contagion law of SC risk evolution. In the complex, dynamic system, the SC risk is highly contagious due to the close correlation between SC nodes.

\subsubsection{The theory of system dynamics}

In 1956, Jay. W. Forrester proposed the system dynamics, a method for systematic analysis of socioeconomic issues [21]. Since then, this method has been extended to various fields from the original application scope, i.e. business management [22]. With the growing awareness of the nonlinearity in society and economy, many have applied the system dynamics to simulate complex socioeconomic systems. The results show that the complex nonlinear systems can be modelled accurately by the system dynamics.

The system dynamics integrates a variety of theories and techniques: feedback control theory, decision theory, test methods for system analysis, system thinking, computer simulation, to name but a few. This integrated method provides an ideal tool to study the SC risk management [23, 24]. After all, the SC risk management requires highly dynamic simulation to capture the time-variation of system behaviour, identify the behavioural changes behind system structure, and optimize structural parameters.

\subsubsection{Modelling of risk contagion in SC}

According to the above hypotheses, the internal risks of the $\mathrm{SC}$ come from the decisions on supply and demand of SC nodes. The decision-making behaviour of each node (enterprise) depends on its ability to satisfy the dynamic demand of consumers in quantity and quality. To fulfil the demand, the enterprise needs to adapt to the market environment, fully understand the market information, and make correct decisions to provide consumers with targeted, quality products and services. Only when the consumer demand is satisfied, can the enterprise mitigate or eliminate SC risk, and maximize its benefits.

The above analysis shows that an SC enterprise needs to control risk contagion considering the system dynamics, complexity and nonlinearity. Thus, the following model was constructed for the internal SC risks per unit time from the perspective of manufacturing enterprises: 


$$
\begin{aligned}
& \frac{d N(t)}{d t}=(1-M) *(1-N(t)) /\left(P_{U} *\left(1-D_{C}\right)\right) \\
& +\left(D_{C} *\left(1-P_{U}\right) * N(t) / Q\right) *(1-N(t))
\end{aligned}
$$

where $P_{U}$ is consumer satisfaction; $D_{C}$ is the consumer churn rate in a production cycle, i.e. the annual mean number of transactions (the value of $D_{C}$ is an integer greater than or equal to zero); $M(0 \leq M \leq 1)$ is the risk immunity of an enterprise; $\mathrm{Q}=\mathrm{g}^{*} \mathrm{H}^{*} \mathrm{~g} /\left(\mathrm{F}^{*} \mathrm{e}^{*} \mathrm{~s}\right)(0<\mathrm{Q} \leq 1)$ is the risk control ability of an enterprise, i.e. the probability of successful handling of SC risk, which was extended from the definition of rod mechanical efficiency. In the formula, $G$ represents the hazard degree of risk, $h$ refers to the number of risks controlled in a period of time, $g$ stands for the probability of successful risk handling in a production cycle (the number of risks controlled / (the total number of risks * the length of the production cycle)), F means the risk handling enthusiasm, e describes the risk control investment, and $\mathrm{s}$ reflects the mean handling duration. $N(t)$ represents the status of enterprise risk in SC. $\frac{d N(t)}{d t}$ shows the contagion effect of risk in SC.

The model shows that the contagion of enterprises in SC is not only related to the risk resistance of enterprise itself, but also to the contagion intensity of risk source.

For simplicity, it is assumed that $Z=(1-M) /\left(P_{G} *\left(1-D_{C}\right)\right)$ and $V=D_{C} *\left(1-P_{U}\right) / Q . Z(0 \leq Z \leq 1)$ is denoted as the coefficient of self-immune risk for an enterprise in SC. This parameter covers consumer satisfaction, consumer churn rate, and the immunity of risk. $\mathrm{V}(0 \leq V \leq 1)$ represents the coefficient of an enterprise's exposure to external risk. The values of $Z$ and $V$ are constants in the same environment, because risk contagion only occurs under specific environment and at specific time. $V$ indicates the effect of risk transmission in SC under certain risk control capability of enterprise. $Z$ shows the effect of risk transmission in supply chain under certain enterprise risk resistance.

Risks will spread from weak to strong and from point to face. Therefore, the assessment of the contagious risk in the budding stage is a key feature in predicting risk. The evolution of risk includes horizontal diffusion and vertical deepening, which is mainly caused by the loss of customers and unsatisfactory customers. Vertical deepening is mainly caused by the enterprise's ability to defend against risks or risk resistance.

\subsubsection{Discussion of this model}

To illustrate the risk contagion characteristics represented by the model, we further decompose this above model. Through the integral operation for equation 1 , the solution is given by formula (2).

$$
N(t)=\frac{\left(1-e^{-(Z+V) t}\right)}{1+\frac{V}{Z} * e^{-(Z+V) t}}
$$

Next, the problem needs to be discussed in three possible cases:

(1) case (I)

After derivative operation of equation 2, then, $\frac{d N(t)}{d t}=\frac{m(Z+V)^{2} e^{-(Z+V) t}}{V\left[1+\frac{Z}{V} e^{-(Z+V) t}\right]^{2}}>0$ it shows that the risk contagion in supply chain will gradually increase with time going forward.

(2) case (II)

$\lim N(t)=1$. It indicates at a certain time the supply

chain risk will be evolved into the active period and will be triggered or even reached a critical point. And then a high-risk period of risk transmission is formed.

(3) case (III)

If $\frac{d^{2} N(t)}{d t^{2}}=0$, then $t=\frac{1}{Z+V} \ln \frac{Z}{V}(\mathrm{t} \geq 0)$. If the value of $t$ is denoted as $T$, then $N(T)=\frac{1}{2}\left(1-\frac{V}{Z}\right)$.

In case (III), if $Z \leq V$, there are no critical point of risk evolution, and the SC risk evolves rapidly first and then slows down; if $\mathrm{Z}>\mathrm{V},\left(\frac{1}{Z+V} \ln \frac{Z}{V}, \frac{1}{2}\left(1-\frac{V}{Z}\right)\right.$ is the critical point of risk evolution. With the increase in the number of spontaneous risk factors, the risk contagion occurs increasingly early.

In order to more intuitively show the relationship between the change of $\mathrm{V}$ and $\mathrm{Z}$ and risk contagion, this paper will simulate the risk contagion effect of Supply Chain Based on MATLAB software.

\section{SIMULATION OF RISK CONTAGION EFFECT}

Computer simulation provides an effective way to study the dynamic changes in complex, nonlinear, high-order systems containing multiple variables and differential equations [25]. MATLAB software as a software of computer simulation, it is the abbreviation of MATRIX LABoratory. It was developed by The MathWorks Company of the United States and can be used for data analysis, data visualization, system simulation, and so on [26]. Because of its powerful function and easy to learn and use, it has been applied in many fields. So, here, based on the software of Matlab 7.1, the risk contagion effect of the model should to be simulated.

To disclose the relationship between risk evolution and parameter change, the values of $\mathrm{Z}$ and $\mathrm{V}$ were kept constant separately during the simulation. In this paper, $0.1,0.4,0.7$ and 0.9 are taken as the values of two parameters respectively to form the simulated risk evolutions in SC system recorded as in Figures 2 and 3 below.

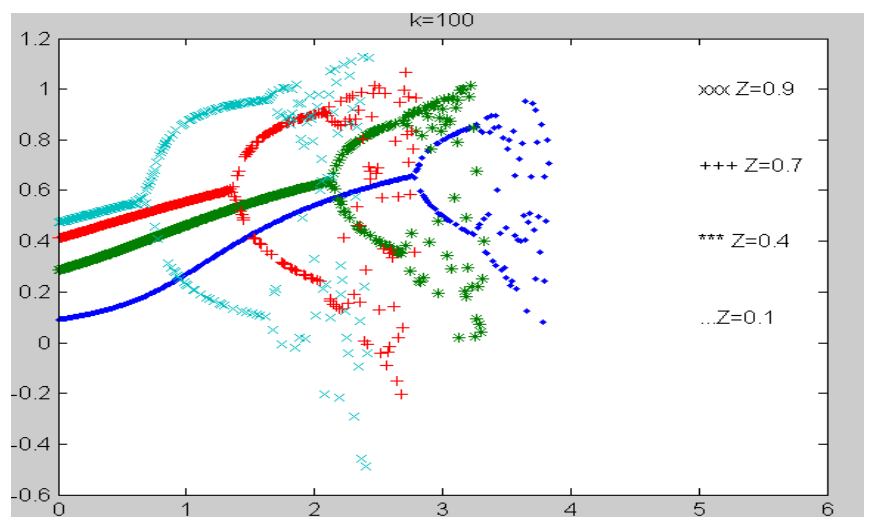

Figure 2. The risk evolution in the SC system under the constant Z 


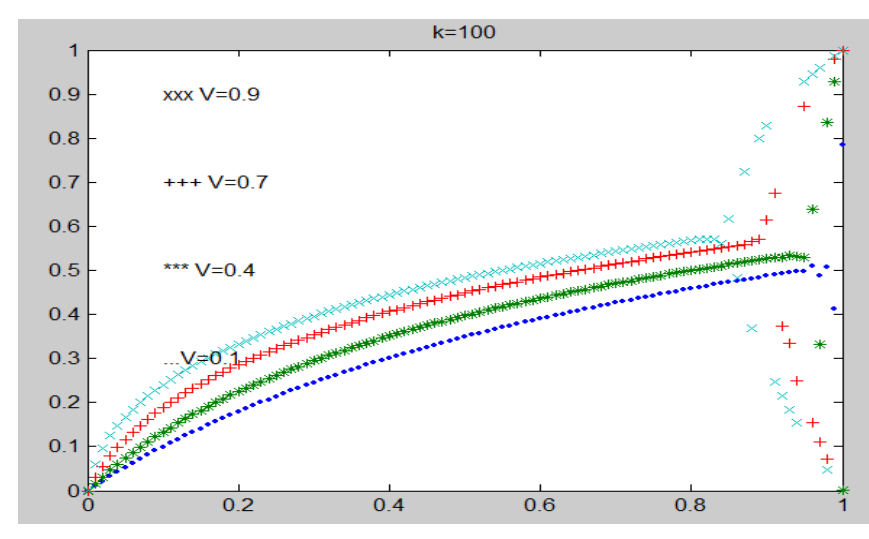

Figure 3. The risk evolution in the SC system under the constant V

As shown in Figures 2 and 3, when either $\mathrm{Z}$ or $\mathrm{V}$ was kept constant, the risk evolution picked up speed with the increase in the other parameter. Thus, the risk evolution speed is positively correlated with $\mathrm{Z}$ value and $\mathrm{V}$ value. It can also be seen from Figure 3 that the greater the $\mathrm{V}$ value, the higher the risk occurrence, and the greater the risk damage.

Next, the risk evolution was simulated respectively under the extreme values of $\mathrm{V}$ and $\mathrm{Z}$. The simulated results are displayed in Figure 4 below.

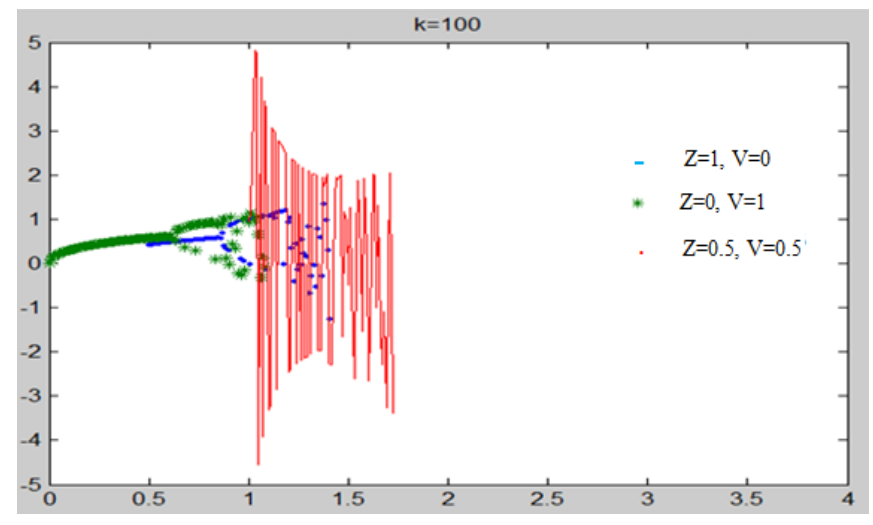

Figure 4. The risk evolution in the SC system under extreme values of $\mathrm{Z}$ and $\mathrm{V}$

As shown in Figure 4, unstable (noisy) the SC system was, the higher the risk, the more obvious the contagion and the more intense the chaos and collision. Specifically, the enterprise with high risk and poor risk resisting ability should start control risk contagion at the $\mathrm{Z}$ value of 0.85 , which indicates the start of chaotic risk evolution in the SC. The enterprise with unobvious risk and moderate risk resistance should begin implementing risk control measures at the $\mathrm{V}$ value of 0.6 . The enterprise with general risk and general risk resistance, which had the widest risk contagion area, should adopt risk control measures at the vertex of the sector of its risk contagion area at the $\mathrm{Z}$ value of 1.4. Thus, different enterprises should make the decision to control SC risk at different times with different strategies. At the same time, assuming that the risk immunity of enterprises is insufficient and the risk control ability of enterprises is strong enough, enterprises will fall into the chaotic and orderly risk contagion and risk control stage at 0.4 risk state. These results have certain reference for enterprises to formulate risk contagion strategies in SC.

In order to further explain the law of SC risk contagion, we classify the enterprise into two cases from the extreme values of $\mathrm{V}$ and $\mathrm{Z}$. on the one hand, the evolution of risk contagion also shows that enterprises with sound risk immunity also have a certain potential risk control ability, which can quickly make adaptive response to delay the contagion of risk. On the other hand, only companies with strong risk control capabilities may not have potential risk immunity. Such enterprises are prone to premature infection risks and will carry out risk control work earlier than the former enterprises. This also reveals that for enterprises in SC, enhancing their risk immunity is far more important than simply emphasizing risk control technology.

Another case for the third kind of enterprise is that presents the component contribution of the two factors on the risk contagion effect of the supply chain. It is learned that the healthier the enterprise, the earlier the risk contagion ended. Thus, healthy enterprises can detect and control risks in time. Incredibly, risk medium- controlling enterprises had the longest risk contagion period among all SC enterprises, indicating that general risk control ability cannot detect and eliminate all SC risks, despite being able to suppress the emergence of same latent risks. The kind of enterprise with general risk resistance and risk control, should to be impacted slowest on by risk sources in SC. Once the risk is introduced, such enterprises will make a great response to adjust their risk immunity and risk control capabilities. Once the panic period is passed, the risk will evolve in infection by an orderly manner. Because these enterprises have certain risk immunity and risk resistance, they often have the worst vigilance against risk contagion.

\section{CONCLUSIONS}

Due to the complexity of risk source and contagion path, based on the analysis of risk contagion process and risk carriers and risk source from consumers of SC regarded as a complex and non-linear system, this paper creatively constructs a risk contagion model based on system dynamics. To test the validity of this model, the Matlab software was applied to simulate the evolution of risk contagion in SC. The simulation results show that the three types of enterprises with different risk resistances have different abilities to control risk contagion, and need to start risk control under different conditions. The study is creative for the explaining of transmission and accumulation of risk for a node in SC evolved into for the whole SC system. It can help enterprises to recognize the crisis they are facing with in risk contagion of SC and the limitations of their ability to cope with risk contagion.

\section{ACKNOWLEDGMENT}

This research was supported by the Natural Science Foundation of Zhejiang Province in China (Grant No.LY18G010011) and supported by Social Science Foundation of Jiaxing University (Grant No.70118001).

\section{REFERENCES}

[1] Cui, B., Wang, Y.X. (2017). Study on guarantee circle risk contagion mechanism in supply chain. Soft Science, 
31(6): 134-138.

[2] Zhang, Y. (2018). The contagion mechanism and measurement of supply chain finance credit risk. A Thesis of Master Degree, Chang'an University.

[3] Xu, M., Yu, D.D. (2016). An empirical analysis on supply chain risk contagion effects. Journal of Xidian University (Social Sciences Edition), 26(2): 13-21.

[4] Zhang, S.Z. (2016). Supply chain risk infection effect analysis considering fairness preference. A Thesis of Master Degree, Chang'an University.

[5] Zhang, S.Z., Pang, C.Y., Li, Q. (2014). Supply chain default risk contagion model. Science and Technology Management Research, (14): 167-170, 175.

[6] Zhou, S.Q., Chang, W.B., Zhou, S.H., Liu, W. (2015). A quantitative model of equipment development quality risk conduction. 2015 Annual Reliability and Maintainability Symposium (RAMS), pp. 1-6. https://doi.org/10.1109/RAMS.2015.7105089

[7] Garvey, M.D., Carnovale, S., Yeniyurt, S. (2015). An analytical framework for supply network risk propagation: A Bayesian network approach. European Journal of Operational Research, 243(2): 618-627. https://doi.org/10.1016/j.ejor.2014.10.034

[8] Tang, L., Jing, K., He, J., Stanley, H.E. (2016). Complex interdependent supply chain networks: cascading failure and robustness. Physica A Statistical Mechanics \& Its Applications, 443: 58-69. https://doi.org/10.1016/j.physa.2015.09.082

[9] Li, Q.M., Shen, L.Y. (2001). Decision-making model incorporating risk behavior under project risk management. System Engineering-Theory \& Practice, 21(10): 1-8.

[10] Zsidisin, G.A. (2003). A grounded definition of supply risk. Journal of Purchasing \& Supply Management, 9(5): 217-224. https://doi.org/10.1016/j.pursup.2003.07.002

[11] Sodhi, M.S., Chopra, S. (2004). Managing risk to avoid supply chain breakdown. MIT Sloan Management Review, 46(1): 53-61.

[12] Chi, Y. (2011). Summary of research on the status and development direction of supply chain risk. Charming China, (15): 16.

[13] Qiu, Y.G. (2010). Research on supply chain risk transmission and its control. Ph.D Dissertation of Wuhan University of Technology.

[14] Li, G. (2011). Research on supply chain risk conduction mechanism. China Business and Market, 25(1): 41-44.

[15] Pang, C.Y. (2015). Study on supply chain default risk contagion model and risk control strategy. Master's Degree Thesis of Chang'an University.

[16] Wang, S.X. (2010). Research on mechanism and control of disruption risk propagation in supply chain networks. Ph.D Dissertation of Donghua University.

[17] Li, G. (2011). Research on supply chain risk conduction mechanism. China Business and Market, 25(1): 41-44.

[18] Wang, J., Wang, X. (2012). Complex dynamic behaviors of constrained supply chain systems. Systems Engineering-Theory \& Practice, 32(4): 746-751. https://dx.chinadoi.cn/10.3969/j.issn.10006788.2012.04.009

[19] Yu, S.H., Chen, G.C. (2016). Research on supply chain models and its dynamical characteristics simulation based on complex system theory. Computer Simulation, 33(4): 328-331, 347.

[20] Wang, D.L., Wang, H.A., Dai, G.Z. (2002). Complexity research of supply chain management. Journal of System Simulation, 14(11): 1439-1442.

[21] Zhong, Y.G., Jia, X.J., Li, X. (2009). System Dynamics. Beijing: Science Press.

[22] Sang, C.X. (2018). Research on the simulation of circular economy in Lanzhou city based on system dynamic. Master's Degree Thesis of Xi'an University of Science and Technology.

[23] Zhang, L.B. (2006). System dynamics based simulation of inventory control in supply chain environment. $\mathrm{PhD}$ Dissertation of Nanjing University of Technology.

[24] Forrester, J.W. (1961). Industrial Dynamics. US, Cambridge Mass: MIT Press.

[25] Richardson, G.P., Pugh, A.L. (1981). Introduction to System Dynamics Modeling. Portland: Productivity Press.

[26] Long, J. (2015). Application of MATLAB in university physics experiment in simulation. The Dissertation of Master Degree, Chang'an University. 